\title{
Evolution of reproductive isolation in plants
}

\author{
A Widmer ${ }^{1}$, C Lexer ${ }^{2}$ and S Cozzolino ${ }^{3}$

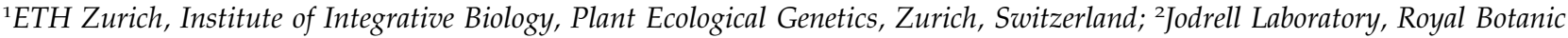 \\ Gardens, Surrey, UK and 'Dipartimento delle Scienze Biologiche, Università degli Studi di Napoli Federico II, Naples, Italy
}

\begin{abstract}
Reproductive isolation is essential for the process of speciation and much has been learned in recent years about the ecology and underlying genetics of reproductive barriers. But plant species are typically isolated not by a single factor, but by a large number of different pre- and postzygotic barriers, and their potentially complex interactions. This phenomenon has often been ignored to date. Recent studies of the relative importance of different isolating barriers between plant species pairs concluded that prezygotic isolation is much stronger than postzygotic isolation. But studies of the patterns of reproductive isolation in plants did not find that prezygotic isolation evolves faster than postzygotic isolation, in contrast to most animals. This may be due to the multiple premating barriers that isolate most species pairs, some of which may be controlled by few genes of major effect and evolve rapidly, whereas others have a
\end{abstract}

complex genetic architecture and evolve more slowly. Intrinsic postzygotic isolation in plants is correlated with genetic divergence, but some instrinsic postzygotic barriers evolve rapidly and are polymorphic within species. Extrinsic postzygotic barriers are rarely included in estimates of different components of reproductive isolation. Much remains to be learned about ecological and molecular interactions among isolating barriers. The role of reinforcement and reproductive character displacement in the evolution of premating barriers is an open topic that deserves further study. At the molecular level, chromosomal and genic isolation factors may be associated and act in concert to mediate reproductive isolation, but their interactions are only starting to be explored.

Heredity (2009) 102, 31-38; doi:10.1038/hdy.2008.69; published online 23 July 2008

Keywords: genic isolation; chromosomal rearrangement; natural selection; prezygotic; postzygotic; speciation

\section{Introduction}

The evolution of reproductive isolation is of central interest in evolutionary biology because of its role in determining gene flow between formerly interbreeding populations (Coyne and Orr, 1998). The last decade has seen great progress in the study of individual components of reproductive isolation among many plant species pairs, including flower color, morphology and odor, flowering phenology or chromosomal divergence (Bradshaw and Schemske, 2003; Marques et al., 2007; Pascarella, 2007; Yang et al., 2007; Waelti et al., 2008), and fascinating insights have recently been obtained into the genetic basis of particular isolating barriers (Wulff et al., 2004; Bomblies et al., 2007; Bomblies and Weigel, 2007; Hoballah et al., 2007). Yet, reproductive isolation among most plant species pairs is not due to a single isolating factor, but is a consequence of a large number of different pre- and postzygotic barriers (Coyne and Orr, 2004; Rieseberg and Willis, 2007), and their potentially complex interactions.

Examples for such interactions between barriers include for example at the ecological level selection on prezygotic barriers due to reduced hybrid viability or fitness upon secondary contact (Dobzhansky, 1937;

Correspondence: $\operatorname{Dr}$ A Widmer, Institute of Integrative Biology, ETH Zurich, Plant Ecological Genetics, Universitätstr. 16, Zurich 8092, Switzerland.

E-mail: alex.widmer@env.ethz.ch

Received 8 February 2008; revised 21 May 2008; accepted 17 June 2008; published online 23 July 2008
Butlin, 1987; Turelli et al., 2001), and at the molecular level the enhancing effects of chromosomal rearrangements on genic isolating barriers due to suppressed recombination (Noor et al., 2001; Rieseberg, 2001; Navarro and Barton, 2003). To date, such interactions have been largely ignored in the plant speciation literature, although understanding complex interactions among different types of isolating barriers in their natural setting is crucial if we want to understand how reproductive isolation evolves.

The major current challenge is thus to identify different isolating barriers and to assess their relative contribution to reproductive isolation, to determine the order and speed with which different barriers arise and to unravel how ecological and molecular interactions among different components of reproductive isolation reduce gene flow among populations and species (Coyne and Orr, 2004).

We searched the recent literature on plant reproductive isolation to look for answers to the following questions: what is the relative importance of prezygotic versus postzygotic barriers in plant reproductive isolation? What do patterns of reproductive isolation in plants look like and are they different from the situation observed in animals? How fast do postzygotic barriers evolve? What is the role of major versus minor genes in premating isolation and are premating barriers reinforced in natural populations? What is the role and molecular basis of trait correlations? What do we know about interactions between genic and chromosomal barriers? 
Here we focus on diploid species and ignore analyses of polyploid species and of diploid-polyploid species pairs, because ploidy differences can lead to unique reproductive barriers between cytotypes (Husband and Sabara, 2004; Kennedy et al., 2006), and allopolyploid speciation has been discussed in detail in recent reviews (Ramsey and Schemske, 1998; Otto and Whitton, 2000; Soltis et al., 2004; Comai, 2005).

\section{Importance of prezygotic versus postzygotic barriers}

Despite the great interest in understanding reproductive isolation, only a small number of studies have investigated the contributions of different isolating barriers to reproductive isolation, and even fewer have attempted to estimate the relative importance of different isolating barriers. Ramsey et al. (2003) investigated different components of reproductive isolation between two sister species of Mimulus, M. cardinalis and M. lewisii that differ in floral traits and attract different pollinators and found that prezygotic isolation more strongly reduced gene flow between species than postzygotic isolation.

Detailed investigation of two closely related neotropical gingers, Costus pulverulents and C. scaber, that are both pollinated by hummingbirds, revealed that prezygotic barriers were strong, resulting in 99-100\% reproductive isolation, depending on the maternal parent, whereas postzygotic barriers were much weaker and their relative contribution to total reproductive isolation was small (Kay, 2006). Similarly, in two hybridizing Penstemon species, Penstemon spectabilis and P. centranthifolius, the bulk of reproductive isolation was inferred to be prezygotic. Pollinator specificity, a prepollination barrier, provided only partial reproductive isolation, but postpollination reproductive isolation mechanisms were substantial. The latter were also found to be highly asymmetric (Chari and Wilson, 2001).

These detailed studies suggest that prezygotic barriers are important for reproductive isolation and pollinators are important, at least in the Mimulus and Costus examples.

Strong premating reproductive isolation can also be brought about by a change in mating system. A further study on Mimulus compared outcrossing $M$. guttatus with selfing M. nasutus (Martin and Willis, 2007). Prezygotic isolation due to a change in mating system was found to contribute much more than postzygotic isolation to total isolation between these species (Martin and Willis, 2007). Given that changes in mating system are common in plants and many plant species are selffertilizing, the evolution of uniparental reproduction may be important in plant reproductive isolation and speciation. An illustrative example is the recent evolution of Senecio eboracensis from Senecio vulgaris. These two species are interfertile and are both visited by generalist pollinators. Because both species are highly selfing, however, hybridization in nature is very rare, which aids the maintenance of species boundaries (Lowe and Abbott, 2004).

All these studies suggest that prezygotic barriers, whether they are brought about by different pollinators, changes in mating system or postmating barriers, are stronger than postzygotic ones, because they more strongly reduce gene flow.

To what extent these studies are representative for flowering plants in general, however, currently remains open. Available evidence may indicate that in some species pairs, postzygotic barriers may sometimes be more important than indicated thus far. In Mediterranean terrestrial orchids, for example, food deceptive species pairs that share a generalized pool of pollinators have significantly more divergent karyotypes compared with species pairs that are pollinated by different insect species, which may indicate that postzygotic barriers conferred by karyotype differences compensate for the weakness of prezygotic barriers in food deceptive orchids (Cozzolino et al., 2004).

An analysis of pre- and postzygotic barriers between sympatric Chamaecrista desvauxii var. graminea and C. desvauxii var. latistipula revealed that flowering periods overlapped for several months and the main pollinator for both varieties was the same bee species, which led the authors to conclude that prezygotic barriers were weak or absent. In contrast, inter-taxa pollinations resulted in no seeds, indicating that postzygotic barriers between taxa are strong (Costa et al., 2007). Similarly, floral isolation between two sympatric Pedicularis species was shown to be incomplete, whereas postzygotic isolation was complete (Yang et al., 2007).

To what extent the relative importance of pre- and postzygotic barriers differ between species with strong premating isolation such as Mimulus (Ramsey et al., 2003; Martin and Willis, 2007), Costus (Kay, 2006) and Penstemon (Chari and Wilson, 2001) on the one hand, and plants that have potentially weaker premating isolation, such as food deceptive orchids (Cozzolino et al., 2004), Chamaecrista taxa (Costa et al., 2007) and Pedicularis species (Yang et al., 2007) on the other hand, however, remains difficult to assess, as long as detailed studies that quantify the relative importance of different isolating barriers are missing for the latter.

Possibly, however, differences will be much smaller than anticipated between plants with specific versus generalist pollinators, once strengths of barriers are calculated in a comparable way.

The insight that prezygotic barriers are stronger than postzygotic ones in the few plant species pairs investigated in sufficient detail to date is based on the approach introduced by Coyne and Orr $(1989,1997)$; Ramsey et al. (2003) that combines estimates of the strength of reproductive isolation from each life-history stage to calculate total isolation and the relative contribution of each isolating barrier. In the final estimates of the relative contribution of each barrier, it is assumed that because in nature, isolating mechanisms act sequentially, each isolating barrier can prevent only the potential gene flow that was not already eliminated by earlier acting barriers. This approach may reflect the relative contribution of each barrier to present day reproductive isolation between species, but may potentially provide a biased perspective on the importance of individual barriers during the evolution of reproductive isolation, and may overestimate the importance of early acting barriers (that is, prezygotic barriers) for the cessation of gene flow between populations and species (Coyne and Orr, 2004). 
For example, only if premating barriers evolve before postmating barriers, will estimates of the relative contribution of premating barriers reflect their importance during the evolution of reproductive isolation. However, it is well established that at least in some instances, postzygotic barriers may evolve within species, before the formation of prezygotic barriers (see below).

Prezygotic barriers may further be overestimated in their contribution to the cessation of gene flow between species because even small amounts of gene flow may be sufficient to prevent primary divergence, at least for neutral loci (Hedrick, 2005), and can lead to strong introgression upon secondary contact (Martin and Willis, 2007; Yatabe et al., 2007). Thus, even though final estimates of the relative cumulative contribution of prezygotic barriers may be over $90 \%$ for outcrossing Mimulus and Costus species pairs (Ramsey et al., 2003; Kay, 2006), and are thus perceived as being the main components ensuring reproductive isolation, the remaining few percentages needed for complete isolation, made up by postzygotic barriers, are essential for the cessation of gene flow between species.

We are not arguing against the importance of prezygotic isolation. It clearly acts as a major filter of interspecific gene flow, but we emphasize that although flowering phenology, pollinator specificity and mating system may be important in isolating many plant species pairs, this barrier is often leaky and 'residual' gene flow overcoming this barrier may be sufficient to amalgamate differentiated populations and species, unless other, postzygotic, barriers are in place.

\section{Patterns of reproductive isolation in plants}

Since the seminal paper of Coyne and Orr (1989), the relationship between reproductive isolation and genetic distance has been investigated in several different animal systems with the general finding that prezygotic isolation evolves faster than postzygotic reproductive isolation (Coyne and Orr, 1989, 1997; Mendelson, 2003). The rate of trait evolution depends on its genetic architecture. Traits that are controlled by few major genes have the potential to evolve faster than traits under the control of numerous genes with minor effects. These insights have led to the perception that in animals, prezygotic barriers are controlled by few major genes and are important during early stages of speciation, whereas postzygotic barriers are controlled by numerous genes of minor effect and accumulate more gradually (Coyne and Orr, 2004).

In contrast to animals, a surprising lack of studies on the patterns of reproductive isolation exists in plants. Moyle et al. (2004) used literature data from three plant genera to analyze two components of postmating reproductive isolation, one prezygotic barrier (success of experimental interspecific pollinations) and one postzygotic barrier (pollen sterility in F1 hybrids). Results varied among genera, ranging from significantly positive (Silene) to weak (Glycine) or no (Streptanthus) correlation between genetic distance and reproductive isolation. For the genera Silene and Glycine (but not Streptanthus), data on both pre- and postzygotic isolation were available and the relative rates of evolution at these two postmating stages were compared. No significant difference was detected in Silene and Glycine, although rate estimates for postzygotic isolation were mostly larger than estimates for prezygotic isolation (Moyle et al., 2004).

A recent experimental comparative study of patterns of reproductive isolation in two orchid groups with different levels of pollinator specificity (Scopece et al., 2007) investigated one premating barrier (pollinator overlap), one postmating prezygotic barrier (fruit production) and a very early postzygotic stage (proportion of viable seeds). No correlation between prezygotic barriers and genetic distance was found for either the premating or postmating barrier, whereas the postzygotic isolation barrier was found to evolve in a clock-like manner in the orchids with overlapping pollinator communities (food deceptive orchids), but not in orchids with highly specific pollination (sexually deceptive orchids).

In a second study on the same groups of orchids, Scopece et al. (2008) found that postzygotic isolation evolved gradually with genetic divergence at each of the three investigated stages, and that late postzygotic isolation (that is, hybrid sterility and hybrid inviability) evolved faster than early postzygotic isolation (that is, embryo mortality). Of the late postzygotic isolation factors, hybrid sterility most likely evolved faster than hybrid inviability. Similar patterns have repeatedly been reported in animals (Wu 1992; Sasa et al., 1998; Presgraves 2002; Price and Bouvier 2002).

The observation made in animals that prezygotic barriers evolve faster than postzygotic ones has thus far not been confirmed in plants (Moyle et al., 2004; Scopece et al., 2007, 2008). Whether this is a consequence of a more complex genetic architecture underlying prezygotic barriers in plants (discussed below) or is due to the fact that in contrast to animals, plants cannot directly choose their mate but depend on often unreliable pollinator service for successful gamete transfer, remains to be tested.

\section{Evolution of postzygotic barriers}

Postzygotic barriers can be separated into intrinsic and extrinsic reproductive isolating barriers. The former includes hybrid inviability and sterility, and the latter ecological and behavioral sterility (Coyne and Orr, 2004).

A common pattern that was found in the studies of Moyle et al. (2004) and Scopece et al. $(2007,2008)$ is that the strength of postzygotic barriers increases with increasing genetic distance among taxa. These studies have focused on intrinsic postzygotic barriers, with the exception of Scopece et al. (2008) where both intrinsic and extrinsic barriers may interact to cause the observed hybrid inviability. However, the experimental design in Scopece et al. (2008) did not allow to disentangle extrinsic and intrinsic causes of hybrid inviability.

The finding that the strength of postzygotic barriers increases with increasing genetic distance may indicate a clock-like and thus slow evolution of postzygotic barriers. However, there is typically considerable variation in the strength of postzygotic isolation, even among recently diverged taxa, indicating that rapid evolution of postzygotic barriers does occur in some groups. In rice, a major crop species, several hybrid sterility and inviability factors have evolved after the separation of indica and japonica subspecies, possibly as consequence of 
domestication (Harushima et al., 2002). In arctic Draba, partial to complete hybrid sterility was found in some intraspecific crosses despite full fertility of the parental plants (Grundt et al., 2006). The sterile, intraspecific hybrids had regular meiosis that makes major chromosomal differences an unlikely cause of the sterility barriers. Instead, a genic basis brought about by Bateson-Dobzhansky-Muller (BDM) incompatibilities was suggested (Grundt et al., 2006). In the model plant species Arabidopsis thaliana, about 2\% of intraspecific crosses give progeny that suffer from hybrid necrosis caused by epistatic interactions among loci and thus by BDM incompatibilities. The identity of involved loci may differ among crosses, but in one case, an allele of an $N B-L R R$ disease resistance gene homolog could be shown to be both necessary and sufficient to cause hybrid necrosis when in combination with a specific allele at a second locus (Bomblies et al., 2007).

Both, A. thaliana and the investigated arctic Draba, are highly selfing which may explain the occurrence and maintenance of strong postzygotic barriers within species, because selfing provides strong premating isolation, thereby facilitating the accumulation of hybrid incompatibilities by genetic drift (Grundt et al., 2006). But intraspecific polymorphisms for hybrid sterility factors are not restricted to highly selfing species. One such factor causing hybrid male sterility in crosses between the outcrossing Mimulus guttatus and the selfing M. nasutus was found to be polymorphic in the outcrossing $M$. guttatus and to be strongly geographically restricted (Sweigart et al., 2007).

These studies highlight that BDM incompatibilities may evolve as intraspecific polymorphisms and can thus appear rapidly when scaled against interspecific species divergence, as in animals (Shuker et al., 2005).

Although we have learned a lot recently about the evolution of intrinsic postzygotic barriers in plants, much less attention has been paid to the role of extrinsic postzygotic barriers in the evolution of plant reproductive isolation. This is surprising, because the study of ecological differences between plant species and of the performance or parental species and their hybrids in different environments has a long history and excellent examples exist (Nilsson, 1983; Wang et al., 1997; Campbell and Waser, 2001; Milne et al., 2003). To date, however, detailed studies that estimate prezygotic isolation together with intrinsic and extrinsic postzygotic isolation are largely missing and so are comparative analyses of the evolution of extrinsic postzygotic isolation in plants.

\section{Genetic basis of premating isolation}

The debate about the role of major genes in adaptation contrasts phenotypic discontinuities with gradual transitions (Orr and Coyne, 1992). Major QTL alleles are expected to generate qualitative differences in phenotype, whereas minor QTL alleles cause a gradation of forms. Numerous QTL studies have suggested that genes of large effect are important in premating isolation between species (Bradshaw and Schemske, 2003; Hoballah et al., 2007), whereas other studies have identified large numbers of relatively minor QTLs underlying premating barriers such as flower phenology, mating system or corolla diameter (Martin et al., 2007;
Martin and Willis, 2007; Moyle, 2007; Lexer and Widmer, 2008). Although these differences make it impossible to settle the debate about the role of major versus minor genes in adaptation and divergence, they could point toward differences in the nature of the adaptive landscape underlying trait divergence. If trait divergence is only possible by a shift between two adaptive peaks that are separated by a valley of low fitness, then trait divergence is most likely due to major genes. In contrast, if adaptive peaks are connected by ridges, trait divergence can also occur gradually and thus be mediated by numerous minor QTLs.

Variation in the number and magnitude of QTL effects may thus reflect variation in the intensity of selection during phenotypic divergence, but it also depends on the genetic architecture of the trait itself that determines its potential for punctuated or more gradual changes. In Petunia, loss-of-function mutations in AN2 that occurred independently more than once caused a shift in flower color from red to white, which induces a major pollinator shift between Petunia integrifolia and P. axillaris. In contrast, differences in the corresponding floral tube morphology appear to be controlled by at least five loci of small to moderate effects, indicating a possible gradual adaptation of the floral tube to optimize pollen transfer by distinct insect visitors (Galliot et al., 2006). Similarly, in Iris fulva and I. brevicaulis, floral trait differences responsible for pollinator preferences are characterized by few QTLs of large effects (Bouck et al., 2007), whereas multiple small effect QTLs are responsible for the difference in flower phenology (Martin et al., 2007).

Although we have learned a lot about the genetic architecture of premating prezygotic barriers, much less is known about the genetics underlying postmating prezygotic barriers, such as male-female interactions during pollen tube reception (Escobar-Restrepo et al., 2007).

\section{Interactions among barriers: ecological and molecular}

We know that plant species are typically separated by multiple reproductive barriers, yet little is known about their interactions, even though the ecological interaction between pre- and postzygotic barriers is a classic problem in evolution (Dobzhansky, 1937).

The phenomenon that direct natural selection strengthens prezygotic isolation to avoid hybridization is predicted to occur upon secondary contact between incipient species that have acquired only partial reproductive isolation in geographic isolation, and is known as reinforcement (Dobzhansky, 1937). The literature about reinforcement is characterized by more debate than firm evidence. A recent study, however, provided compelling evidence for reinforcement of a flowering time shift in the grass Anthoxanthum odoratum (Silvertown et al., 2005), and a macroevolutionary analysis of the Cape Flora of South Africa suggested that pollinator shifts can occur upon secondary contact to prevent gene flow between incipient species that initially diverged as a consequence of adaptation to other ecological conditions, such as soil composition. In this context, the enhancement of reproductive isolation by 
pollinator shifts is thought to occur as a result of selection against unfit hybrids, and thus as a consequence of reinforcement (Van der Niet et al., 2006).

While reinforcement is thought to complete the speciation process between incipient species upon secondary contact by strengthening prezygotic barriers to halt gene flow, reproductive character displacement (sensu Butlin, 1987) occurs as a consequence of interactions in sympatry among reproductively isolated species, and serves to reduce gamete loss in unsuccessful heterospecific matings. Reproductive character displacement may be a plausible explanation for the evolution of premating barriers when postzygotically strongly isolated species come into contact, because selection against hybrids is maximal and the process cannot be halted by recombination, in contrast to reinforcement. It may thus help to explain the observation that plant groups characterized by strong and often complete postzygotic isolation have also evolved a considerable amount of prezygotic isolation. Few studies have found convincing evidence for reproductive character displacement between sympatric, congeneric plant species (Armbruster et al., 1994; Muchhala and Potts, 2007), and to the best of our knowledge, none of these studies have related reproductive character displacement to the strength of postzygotic reproductive isolation. Thus, much remains to be learned about ecological interactions among premating and postmating reproductive barriers, and the order of their evolution.

Interactions among barriers need not be mediated by ecology but can also have a molecular basis. Correlations among phenotypic traits are commonly observed in both animals and plants. Trait correlations can occur as a consequence of natural selection and thus have an ecological basis, or as a consequence of either pleiotropy or close linkage and thus have a molecular basis. If loci are further apart on chromosomes, suppression of recombination, for example mediated by chromosomal inversions or reduced recombination on sex chromosomes, can maintain trait correlations and lead to the formation of co-adapted gene complexes (Dobzhansky, 1970; Coyne and Orr, 2004).

QTL analyses of traits potentially involved in pre- or postzygotic isolating barriers in plants have repeatedly found that QTLs for different traits cluster in particular areas of the genome (Fishman et al., 2002; Hodges et al., 2002; Rieseberg et al., 2003; Bratteler et al., 2006; Goodwillie et al., 2006; Moyle, 2007), thus providing evidence for the occurrence of pleiotropy or linkage among QTLs for different traits. Whether such clustering of QTLs is due to pleiotropy or tight linkage is often unknown (Conner, 2002), but both cause strong correlations among the traits involved. Selection on any one of the correlated traits can then induce correlational selection on other traits, which has the potential to lead to trait divergence also in traits that are not the direct target of selection, and may provide a molecular basis for reproductive character displacement or reinforcement.

\section{Interactions among chromosomal and genic isolating barriers}

Chromosomal rearrangements have long been recognized as important isolating barriers in plants (White,
1978; Levin, 2002). Whereas in most classical models of chromosomal speciation, rearrangements act as isolating barriers because of their intrinsic effects on hybrid fitness, a more recent hypothesis is that they do so by indirect effects on genic isolating barriers. Chromosomal rearrangements may increase the strength of genic barriers either by suppressing recombination and thus extending the effects of linked isolation genes over increased chromosomal distances, or by an effective reduction in recombination due to selection against recombinant gametes (Rieseberg, 2001). Thus, chromosomal rearrangements may favor the accumulation of genic postzygotic barriers involving locally adapted alleles or epistatic (BDM) incompatibilities in parapatry (Navarro and Barton, 2003).

Recent experimental results from animals and plants support an important role for chromosomal rearrangements in speciation. In Drosophila, inversions are more frequently found between recently diverged sympatric species pairs than among recently diverged allopatric species, consistent with a rearrangement-mediated reduction in gene flow in sympatry or parapatry (Noor et al., 2001). In Helianthus, introgression across interspecific hybrid zones is greatly reduced in rearranged compared to collinear regions of the genome (Rieseberg et al., 1999). In Mediterranean orchids, rearrangements are more likely to be found between closely related species that share pollinators compared to species pairs with very specific pollination syndromes (Cozzolino et al., 2004). All of these results support a role for rearrangements in the origin or maintenance of reproductive isolation, but it is notoriously difficult to disentangle the direct effects of rearrangements from their indirect effects on genic barriers by the suppression of recombination. Recent results from Helianthus are partially informative in this respect.

Rearrangements appear to have been important during diploid hybrid speciation in Helianthus (Rieseberg et al., 1995). Whereas recent modeling work suggests that much of the sunflower genome must have experienced recombination for hundreds of generations before genome stabilization was complete (Buerkle and Rieseberg, 2007), the low-recombination portions of chromosomes that went to fixation early on during the speciation process are yet to be identified. Rearrangements also contribute to the maintenance of reproductive isolation when parapatric sunflower species such as Helianthus annuus and $H$. petiolaris come into secondary contact (Rieseberg et al., 1999). Rearrangements between $H$. annuus and $H$. petiolaris accumulate at a rate of 5.5-7.3 per million years, the highest rate reported for any taxonomic group to date (Burke et al., 2004). Two observations support a role for rearrangements in the accumulation of genic isolating barriers between these two species. First, several of the QTL conferring pollen sterility, an important intrinsic isolating barrier, map to collinear regions of the Helianthus genome, consistent with the action of genic isolation factors in addition to chromosomal ones (Rieseberg et al., 1999). Second, epistatic interactions among sterility QTL that do map to chromosomal rearrangements generally do not involve linkage groups that are part of the same multivalent configuration during meiosis (Lai et al., 2005). Both observations suggest that the effects of rearrangements on reproductive isolation are not due to their 
direct effects on fertility alone but may also involve indirect effects by genic isolating barriers.

More conclusive evidence for or against a role of rearrangements in the accumulation of isolation genes may be obtained by studying the molecular genetic signature of positive selection in rearranged and collinear genomic regions (Navarro and Barton, 2003). Such tests are within reach in a rapidly growing number of taxonomic groups with existing genome programs (Noor and Feder, 2006). In the case of inversions, future studies should also address the question of whether rearrangements actually caused the accumulation of genic isolating barriers or whether the sequence of events was reversed. The latter may be the case when inversions 'capture' already locally adapted alleles and subsequently spread by reducing recombination, a hypothesis that may be tested by determining the age of selected mutations and inversion breakpoints (Kirkpatrick and Barton, 2006).

\section{Conclusions}

The evolution of reproductive isolation is a classic problem in evolutionary biology. Much progress has been made in recent years in the analysis and characterization of individual components of reproductive isolation, but much remains to be learned about the sequence of evolution of pre- and postzygotic barriers, about their ecological interactions, and about the genetic architecture of reproductive isolation. It is clear that studies of individual components of reproductive isolation are needed to further our understanding of the processes and mechanisms underlying isolation factors, but to understand reproductive isolation, it is essential that the effects of isolating factors are investigated in combination with other barriers.

The enigmatic process of speciation is nowadays often equated with the evolution of reproductive isolation, although it is unclear how much reproductive isolation is required for speciation to occur. To provide an example, recent data on homoploid hybrid or 'recombinational' speciation suggest that the role of reproductive isolation relative to genome stabilization in this hotly debated speciation mode has been greatly underestimated previously (Buerkle and Rieseberg, 2007). On a more general level, ecological speciation often is thought to proceed in situations of divergence with gene flow (that is, in parapatry) and great potential for the accumulation of genetic differences through drift (Schluter, 2000), for example, in clinal or stepping-stone models of speciation (Coyne and Orr, 2004). The relative roles of geographic isolation vs selection associated with reproductive isolation to complete speciation in such cases are little understood.

It seems clear that speciation is unlikely to be a consequence of a single isolating barrier or a single gene, at least in plants. Fortunately, many plant species are able to form viable hybrids and many hybrid combinations can be found in (semi-)natural populations. Such hybrid zones provide a natural arena in which individual components of reproductive isolation and interactions among isolating barriers can be investigated, because recombination breaks up associations not only among traits, but also among interacting genes. Thus, hybrid zones can serve as 'evolutionary genomics' laboratories for studying the genetic basis of multiple different reproductive barriers in situ (Noor and Feder, 2006), except for very strongly selected barriers acting early on during development. Genetic variants associated with such barriers will not be visible in hybrid zones because they will be eliminated by selection. Hence, it seems likely that combinations of this naturalist 'speciation genomics' approach and more reductionist experiments in laboratory settings will be the key to understanding the origin and evolution of complex reproductive barriers in plants. Furthermore, it is essential that studies on plant reproductive isolation are not limited to analyses that can be performed in the laboratory or greenhouse, because entire groups of isolating barriers, such as extrinsic postzygotic barriers, would otherwise be missed. Instead, the study of plant reproductive isolation requires the integration of population and functional genomic studies in the laboratory with greenhouse experiments, field observations and reciprocal transplant experiments.

\section{Acknowledgements}

We are grateful to Loren $\mathrm{H}$ Rieseberg for transplanting his enthusiasm for plant speciation into our souls and for providing us with the great opportunity to meet many years ago and start working on plant speciation in his laboratory at Indiana University, and at a Steak house in downtown Bloomington where we spent long winter evenings arguing about plant speciation and eating loads of meat to recover from the vegetarian lunch meetings with the master.

\section{References}

Armbruster WS, Edwards ME, Debevec EM (1994). Floral character displacement generates assemblage structure of Western-Australian Triggerplants (Stylidium). Ecology 75: 315-329.

Bomblies K, Lempe J, Epple P, Warthmann N, Lanz C, Dangl JL et al. (2007). Autoimmune response as a mechanism for a Dobzhansky-Muller-type incompatibility syndrome in plants. PLoS Biol 5: 1962-1972.

Bomblies K, Weigel D (2007). Hybrid necrosis: autoimmunity as a potential gene-flow barrier in plant species. Nat Rev Genet 8: 382-393.

Bouck A, Wessler SR, Arnold ML (2007). Qtl analysis of floral traits in Louisiana iris hybrids. Evolution 61: 2308-2319.

Bradshaw HD, Schemske DW (2003). Allele substitution at a flower colour locus produces a pollinator shift in monkeyflowers. Nature 426: 176-178.

Bratteler M, Lexer C, Widmer A (2006). Genetic architecture of traits associated with serpentine adaptation of Silene vulgaris. J Evol Biol 19: 1149-1156.

Buerkle C, Rieseberg LH (2007). The rate of genome stabilization in homoploid hybrid species. Evolution 62: 266-275.

Burke JM, Lai Z, Salmaso M, Nakazato T, Tang SX, Heesacker A et al. (2004). Comparative mapping and rapid karyotypic evolution in the genus Helianthus. Genetics 167: 449-457.

Butlin R (1987). Speciation by reinforcement. Trends Ecol Evol 2: $8-13$.

Campbell DR, Waser NM (2001). Genotype-by-environment interaction and the fitness of plant hybrids in the wild. Evolution 55: 669-676.

Chari J, Wilson P (2001). Factors limiting hybridization between Penstemon spectabilis and Penstemon centranthifolius. Can J Bot 79: 1439-1448. 
Comai L (2005). The advantages and disadvantages of being polyploid. Nat Rev Genet 6: 836-846.

Conner JK (2002). Genetic mechanisms of floral trait correlations in a natural population. Nature 420: 407-410.

Costa CBN, Lambert SM, Borba EL, De Queiroz LP (2007). Postzygotic reproductive isolation between sympatric taxa in the Chamaecrista desvauxii complex (Leguminosae-Caesalpinioideae). Ann Bot 99: 625-635.

Coyne JA, Orr HA (1989). Patterns of speciation in Drosophila. Evolution 43: 362-381.

Coyne JA, Orr HA (1997). 'Patterns of speciation in Drosophila' revisited. Evolution 51: 295-303.

Coyne JA, Orr HA (1998). The evolutionary genetics of speciation. Philos Trans $R$ Soc Lond B Biol Sci 353: 287-305.

Coyne JA, Orr HA (2004). Speciation. Sinauer Associates: Sunderland, MA.

Cozzolino S, D'Emerico S, Widmer A (2004). Evidence for reproductive isolate selection in Mediterranean orchids: karyotype differences compensate for the lack of pollinator specificity. Proc R Soc Lond B Biol Sci 271: S259-S262.

Dobzhansky T (1937). Genetics and the Origin of Species. Columbia University Press: New York, NY.

Dobzhansky T (1970). Genetics of the Evolutionary Process. Columbia University Press: NY.

Escobar-Restrepo JM, Huck N, Kessler S, Gagliardini V, Gheyselinck J, Yang WC, et al. (2007). The FERONIA receptor-like kinase mediates male-female interactions during pollen tube reception. Science 317: 656-660.

Fishman L, Kelly AJ, Willis JH (2002). Minor quantitative trait loci underlie floral traits associated with mating system divergence in Mimulus. Evolution 56: 2138-2155.

Galliot C, Hoballah ME, Kuhlemeier C, Stuurman J (2006). Genetics of flower size and nectar volume in Petunia pollination syndromes. Planta 225: 203-212.

Goodwillie C, Ritland C, Ritland K (2006). The genetic basis of floral traits associated with mating system evolution in Leptosiphon (Polemoniaceae): an analysis of quantitative trait loci. Evolution 60: 491-504.

Grundt HH, Kjolner S, Borgen L, Rieseberg LH, Brochmann C (2006). High biological species diversity in the arctic flora. Proc Natl Acad Sci USA 103: 972-975.

Harushima Y, Nakagahra M, Yano M, Sasaki T, Kurata N (2002). Diverse variation of reproductive barriers in three intraspecific rice crosses. Genetics 160: 313-322.

Hedrick PW (2005). Genetics of Populations, 3rd edn. Jones and Bartlett Publishers: Sudbury, MA.

Hoballah ME, Gubitz T, Stuurman J, Broger L, Barone M, Mandel T, et al. (2007). Single gene-mediated shift in pollinator attraction in Petunia. Plant Cell 19: 779-790.

Hodges SA, Whittall JB, Fulton M, Yang JY (2002). Genetics of floral traits influencing reproductive isolation between Aquilegia formosa and Aquilegia pubescens. Am Nat 159: S51-S60.

Husband BC, Sabara HA (2004). Reproductive isolation between autotetraploids and their diploid progenitors in fireweed, Chamerion angustifolium (Onagraceae). New Phytol 161: 703-713.

Kay KM (2006). Reproductive isolation between two closely related hummingbird-pollinated neotropical gingers. Evolution 60: 538-552.

Kennedy BF, Sabara HA, Haydon D, Husband BC (2006). Pollinator-mediated assortative mating in mixed ploidy populations of Chamerion angustifolium (Onagraceae). Oecologia 150: 398-408.

Kirkpatrick M, Barton N (2006). Chromosome inversions, local adaptation and speciation. Genetics 173: 419-434.

Lai Z, Nakazato T, Salmaso M, Burke JM, Tang SX, Knapp SJ et al. (2005). Extensive chromosomal repatterning and the evolution of sterility barriers in hybrid sunflower species. Genetics 171: 291-303.

Levin D (2002). The Role of Chromosomal Change in Plant Evolution. Oxford University Press: Oxford.
Lexer C, Widmer A (2008). The genic view of plant speciationrecent progress and emerging questions. Philos Trans $R$ Soc Lond B Biol Sci.

Lowe AJ, Abbott RJ (2004). Reproductive isolation of a new hybrid species, Senecio eboracensis Abbott \& Lowe (Asteraceae). Heredity 92: 386-395.

Marques I, Rossello-Graell A, Draper D, Iriond JM (2007). Pollination patterns limit hybridization between two sympatric species of Narcissus (Amaryllidaceae). Am J Bot 94: 1352-1359.

Martin NH, Bouck AC, Arnold ML (2007). The genetic architecture of reproductive isolation in Louisiana irises: flowering phenology. Genetics 175: 1803-1812.

Martin NH, Willis JH (2007). Ecological divergence associated with mating system causes nearly complete reproductive isolation between sympatric Mimulus species. Evolution 61: 68-82.

Mendelson TC (2003). Sexual isolation evolves faster than hybrid inviability in a diverse and sexually dimorphic genus of fish (Percidae: Etheostoma). Evolution 57: 317-327.

Milne RI, Terzioglu S, Abbott RJ (2003). A hybrid zone dominated by fertile $\mathrm{F}(1) \mathrm{s}$ : maintenance of species barriers in Rhododendron. Mol Ecol 12: 2719-2729.

Moyle LC (2007). Comparative genetics of potential prezygotic and postzygotic isolating barriers in a lycopersicon species cross. J Hered 98: 123-135.

Moyle LC, Olson MS, Tiffin P (2004). Patterns of reproductive isolation in three angiosperm genera. Evolution 58: 1195-1208.

Muchhala N, Potts MD (2007). Character displacement among bat-pollinated flowers of the genus Burmeistera: analysis of mechanism, process and pattern. Proc $R$ Soc Lond B Biol Sci 274: 2731-2737.

Barton NH (2003). Accumulating postzygotic isolation genes in parapatry: a new twist on chromosomal speciation. Evolution 57: 447-459.

Nilsson LA (1983). Processes of isolation and introgressive interplay between Platanthera bifolia (L) rich and Platanthera chlorantha (Custer) Reichb (Orchidaceae). Bot J Linn Soc 87: 325-350.

Noor MAF, Feder JL (2006). Speciation genetics: evolving approaches. Nat Rev Genet 7: 851-861.

Noor MAF, Grams KL, Bertucci LA, Reiland J (2001). Chromosomal inversions and the reproductive isolation of species. Proc Natl Acad Sci USA 98: 12084-12088.

Orr HA, Coyne JA (1992). The genetics of adaptation-a reassessment. Am Nat 140: 725-742.

Otto SP, Whitton J (2000). Polyploid incidence and evolution. Annu Rev Genet 34: 401-437.

Pascarella JB (2007). Mechanisms of prezygotic reproductive isolation between two sympatric species, Gelsemium rankinii and $G$. sempervirens (Gelsemiaceae), in the southeastern United States. Am J Bot 94: 468-476.

Presgraves DC (2002). Patterns of postzygotic isolation in Lepidoptera. Evolution 56: 1168-1183.

Price TD, Bouvier MM (2002). The evolution of $F_{1}$ postzygotic incompatibilities in birds. Evolution 56: 2083-2089.

Ramsey J, Bradshaw HD, Schemske DW (2003). Components of reproductive isolation between the monkeyflowers Mimulus lewisii and M. cardinalis (Phrymaceae). Evolution 57: 1520-1534.

Ramsey J, Schemske DW (1998). Pathways, mechanisms, and rates of polyploid formation in flowering plants. Annu Rev Ecol Syst 29: 467-501.

Rieseberg LH (2001). Chromosomal rearrangements and speciation. Trends Ecol Evol 16: 351-358.

Rieseberg LH, Linder CR, Seiler GJ (1995). Chromosomal and genic barriers to introgression in Helianthus. Genetics 141: $1163-1171$.

Rieseberg LH, Raymond O, Rosenthal DM, Lai Z, Livingstone K, Nakazato T et al. (2003). Major ecological transitions in 
wild sunflowers facilitated by hybridization. Science 301 1211-1216.

Rieseberg LH, Whitton J, Gardner K (1999). Hybrid zones and the genetic architecture of a barrier to gene flow between two sunflower species. Genetics 152: 713-727.

Rieseberg LH, Willis JH (2007). Plant speciation. Science 317: 910-914.

Sasa MM, Chippindale PT, Johnson NA (1998). Patterns of postzygotic isolation in frogs. Evolution 52: 1811-1820.

Schluter D (2000). The Ecology of Adaptive Radiation. Oxford University Press: Oxford.

Scopece G, Musacchio A, Widmer A, Cozzolino S (2007). Patterns of reproductive isolation in Mediterranean deceptive orchids. Evolution 61: 2623-2642.

Scopece G, Widmer A, Cozzolino S (2008). Evolution of postzygotic reproductive isolation in a guild of deceptive orchids. Am Nat 171: 315-326.

Shuker DM, Underwood K, King TM, Butlin RK (2005). Patterns of male sterility in a grasshopper hybrid zone imply accumulation of hybrid incompatibilities without selection. Proc R Soc Lond B Biol Sci 272: 2491-2497.

Silvertown J, Servaes C, Biss P, Macleod D (2005). Reinforcement of reproductive isolation between adjacent populations in the Park Grass Experiment. Heredity 95: 198-205.

Soltis DE, Soltis PS, Tate JA (2004). Advances in the study of polyploidy since plant speciation. New Phytol 161: 173-191.

Sweigart AL, Mason AR, Willis JH (2007). Natural variation for a hybrid incompatibility between two species of Mimulus. Evolution 61: 141-151.
Turelli M, Barton NH, Coyne JA (2001). Theory and speciation. Trends Ecol Evol 16: 330-343.

Van der Niet T, Johnson SD, Linder HP (2006). Macroevolutionary data suggest a role for reinforcement in pollination system shifts. Evolution 60: 1596-1601.

Waelti MO, Muhlemann JK, Widmer A, Schiestl FP (2008). Floral odour and reproductive isolation in two species of Silene. J Evol Biol 21: 111-121.

Wang H, McArthur ED, Sanderson SC, Graham JH, Freeman DC (1997). Narrow hybrid zone between two subspecies of big sagebrush (Artemisia tridentata: Asteraceae) 4. Reciprocal transplant experiments. Evolution 51: 95-102.

White M (1978). Modes of Speciation. W.H. Freeman \& Co.: San Francisco, CA.

Wu CI (1992). A note on Haldane's rule: hybrid inviability versus hybrid sterility. Evolution 46: 1584-1587.

Wulff BBH, Kruijt M, Collins PL, Thomas CM, Ludwig AA, De Wit PJGM, et al. (2004). Gene shuffling-generated and natural variants of the tomato resistance gene Cf-9 exhibit different auto-necrosis-inducing activities in Nicotiana species. Plant $J$ 40: 942-956.

Yang CF, Gituru RW, Guo YH (2007). Reproductive isolation of two sympatric louseworts, Pedicularis rhinanthoides and Pedicularis longiflora (Orobanchaceae): how does the same pollinator type avoid interspecific pollen transfer? Biol J Linn Soc Lond 90: 37-48.

Yatabe Y, Kane NC, Scotti-Saintagne C, Rieseberg LH (2007). Rampant gene exchange across a strong reproductive barrier between the annual sunflowers, Helianthus annuus and H. petiolaris. Genetics 175: 1883-1893. 